\title{
Convoy driving through ad-hoc coalition formation
}

\author{
Majid Ali Khan and Ladislau Bölöni \\ Department of Electrical and Computer Engineering \\ University of Central Florida \\ Orlando, Florida, 32816 \\ Email: khan@bond.cs.ucf.edu, lboloni@cpe.ucf.edu
}

\begin{abstract}
Convoy driving on public highways is a useful phenomena which increases the safety and the throughput of the highway. We present an approach through which a wireless Convoy Driving Device assists the driver in the task of deciding to join or leave a convoy, influencing the speed and formation of the convoy. Our approach handles complex situations like the merging and splitting of convoys, and it offers valuable lessons with applications for other cases of teamwork of mobile entities.
\end{abstract}

\section{PROBLEM DESCRIPTION}

Convoy driving on a public highway is a phenomena with multiple benefits. It makes driving safer, it increases the throughput of the highway and decreases travel time. As highway traffic is often modelled by analogies with fluid dynamics [1], in this language convoy driving corresponds to a flow without turbulences.

While the formation of convoys is sometimes an explicitly planned operation, most often it is happening in an ad-hoc manner between vehicles whose drivers do not know each other, might not have common goals and can communicate only through indirect means. Convoys are formed and dissolved dynamically, their lifecycle ranges from tens of seconds to several hours. Vehicles can join and leave, and convoys themselves can split and merge.

If we consider the vehicles governed by intelligent agents, highway convoy driving is a microcosm of problems including communication (both at networking and semantic level), team formation, leader election, negotiation and planning. Being a one-dimensional world, highway driving is a particular case in which these techniques and theories can be tested. Similar techniques can then be applied to two-dimensions (for example unmanned ground vehicles) and three-dimensions (e.g. unmanned aerial vehicles). However, far from being a toy problem, convoy formation in highway driving is an economically important problem in its own and it presents specific challenges not present in the two or three dimensional case. One example is the difficulty to overtake vehicles or the influence of traffic signs.

In this paper we present a set of algorithms designed and implemented to facilitate convoy formation on the highway. Assuming that highway vehicles are completely under agent control is unrealistic at this stage of the technology. Therefore, we consider the vehicles to be controlled by the human driver who is assisted by an additional "convoy driving device" (CDD). The CDD is a hardware device, with limited computational power and low power wireless communication abilities.
We assume that it can read the speed of the vehicle, and it might be able to determine the distance from the vehicle in front. Its output is a "accelerate" and "deccelerate" message, which is conveyed to the driver through visual means. We can envision implementations in which these signals are connected to the "increase speed" / "decrease speed" controls of the cruise control of the vehicle which is the case of the Adaptive Cruise Control devices proposed by some automakers.

The CDD-s are communicating with each other, and decide on the formation of a convoy based on a variety of factors such as the current speed of the vehicles, the desired speed, the limitations of the vehicles and highway speed limits. The common speed of the convoy is negotiated, and the vehicles adjust their speed such that the convoy is maintained stable, preferably with uniform following distances. The algorithms need to handle a variety of events in the lifecycle of the convoy: vehicles joining and leaving the convoy, convoys with different speeds passing by each other, the vehicles in the convoy separating in two different convoys with different speeds or two convoys merging into a single one.

We have physically implemented the CDD by using "motes", tiny, self-contained, battery powered computers with sensor and wireless capabilities [2]. These devices are a relatively good match to our assumption about the CDD. We need to emphasize however, that a potential commercial implementation of a CDD would likely use hardware with different characteristics, potentially more powerful and better integrated with the rest of the on-board electronics.

We used our hardware implementation to test the sensing and inter-device communication. We used computer simulation to test the various coalition formation algorithms.

The remainder of this paper is organized as follows. Section II explains our coalition formation approach, Section III discusses the algorithms used for coalition formation and evaluation of the relative utility of coalitions. Section IV discusses the hardware implementation of the Convoy Driving Device based on the mote architecture. A series of simulation studies comparing variants of the coalition formation algorithms under realistic scenarios are presented in Section V. We overview related work in Section VI and conclude in Section VII.

\section{COALITION FORMATION}

Let us consider a set of vehicles $V=\{v 1, v 2, \ldots, v n\}$ moving in the same direction on a highway. We call a coalition $C \subseteq V$, a set of vehicles which are moving in a coordinated speed and close proximity to each other. 
A coalition among disparate nodes can be formed by either a centralized or a distributed approach. In a centralized approach, a coalition leader is selected which can define the structure of the coalition based on available global knowledge of the network. Such a global knowledge can be acquired by devising a message passing scheme between nodes. A multihop routing scheme might also be required when the coalition leader is not directly connected with each participating node. Such a centralized scheme, however, does not scale well in a highly dynamic environment with a large number of nodes. The reason being that it becomes quite cumbersome to obtain global knowledge and to determine coalition leaders in such a large dynamic environment.

In a distributed approach, however, every node can be made autonomous. Nodes decide their respective coalitions based on local neighbourhood information. Overall coalition structure, therefore, evolves out of these individual decisions. Since no centralized control is required, there is no need to elect a coalition leader. This kind of distributed approach usually scales well in a large dynamic environment. Since vehicles movement on a highway is a highly dynamic environment with large number of nodes, we chose to take a distributed approach towards the problem. In the next section, we present a distributed coalition formation algorithm, which will be used by each participating node of the network.

\section{COALITION FORMATION ALGORITHM}

The simplest way to create a convoy is for every vehicle to adopt the speed of the front vehicle. For example the Adaptive Cruise Control systems tested by various vehicle manufacturers are working like this. This approach however, has several drawbacks. The speed of the convoy will be dictated by its slowest member. If any vehicle decides to go slower than the vehicle in front, it will lead to splitting of the convoy.

By allowing the agents controlling the vehicles to form a coalition through wireless ad-hoc networking, we are able to create a more efficient organization. There are three different aspects of the participation of a vehicle in a convoy.

- The decision to join or leave the convoy. The vehicle can join any convoy in its physical proximity, or it can decide to drive outside of any convoy. For the sake of a uniform treatment, we will consider this case as the vehicle forming its own convoy. These decisions are based by the vehicle evaluating the utility of the convoy and at the same time considering the costs associated with joining and leaving the convoys. We note that the utility of a convoy depends on the preferences and capabilities of the vehicle, and it can vary in time as parameters change.

- The influence of the convoy on the vehicle. Once the vehicle has joined the convoy, its driving is influenced by the presence of the other vehicles in the convoy. Most obviously, its speed needs to be synchronized with the speed of the other vehicles. Small, temporary adjustments in speed can be used to achieve the desired following distance / time gap between the vehicles.
- The influence of the vehicle on the convoy. The vehicle should be able to influence the parameters of the convoy such as speed and following distance through a process of negotiating with the other agents participating in the coalition.

In order to accomplish these goals, the agent contains two, largely independent components. The performance evaluation component (Figure $1 \mathrm{a}$ ) continuously evaluates the utility of the current coalition. The coalition join decision component (Figure $1 \mathrm{~b}$ ) is responsible for making decisions about which coalitions will the agent join or leave based on "offered" coalitions. These two components operate continuously and in parallel, such that the coalition join decisions are always made based on the latest available data.

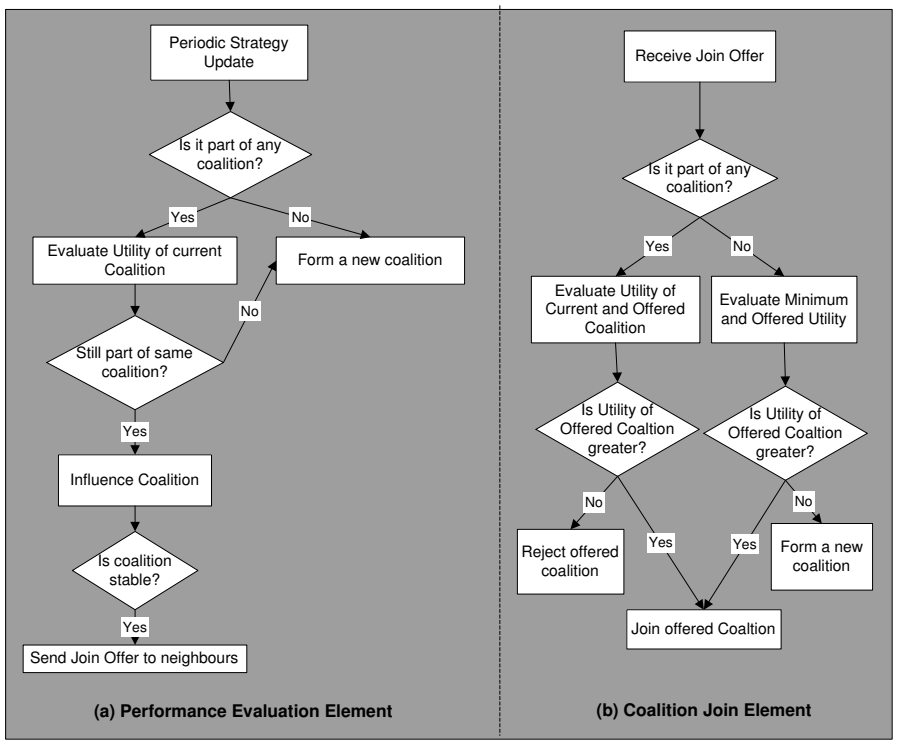

Fig. 1. Coalition formation: Performance element of agent

Before discussing these algorithms in detail, we need to introduce several notations:

$V i: \quad$ a vehicle $i$

$C i$ : $\quad$ coalition identifier for vehicle $i$

$N i: \quad$ set of vehicles in the neighbourhood of vehicle $i$

$U[i, C i]: \quad$ utility of vehicle $i$ for being in coalition $C i$

$M i: \quad$ minimum acceptable utility for vehicle $i$

Sij: $\quad$ speed offered to vehicle $i$ by coalition $C j$

Each coalition is identified by a unique positive coalition identifier. We will further assume that each agent knows about the vehicles in its neighbourhood and keeps updating it on periodic basis, by using a simple beacon approach.

In the performance evaluation algorithm, each agent will periodically check if it is part of any coalition, i.e. whether its coalition identifier, $\mathrm{C} i$, is some positive number. If it is not, then it will create its own coalition identifier using formSelfCoalition() method. However, if it is part of an existing coalition, it will evaluate the utility of this coali- 

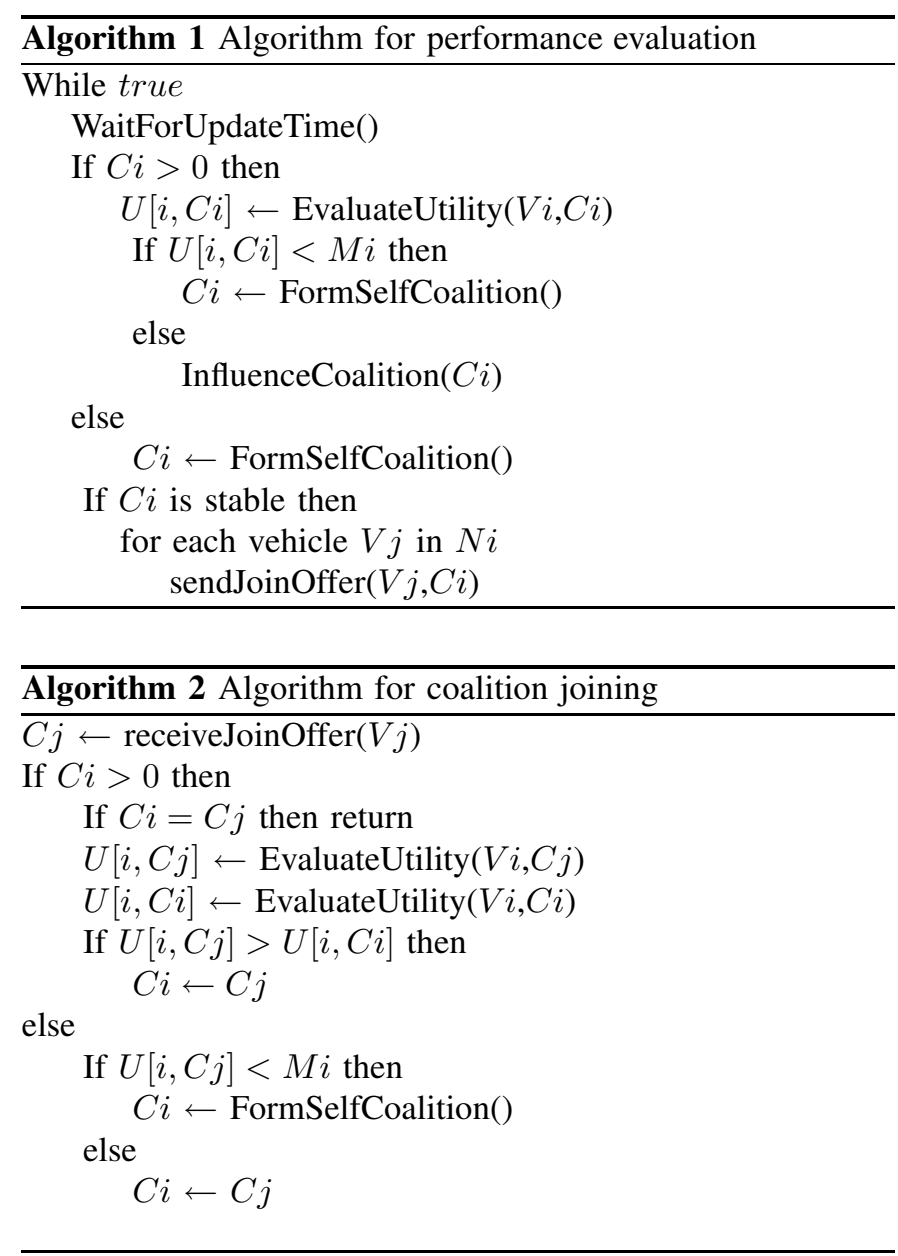

tion using evaluateUtility() method. If the utility $U[i, C i]$ is larger than its minimum acceptable utility $M i$, it will stay in the coalition. Otherwise, it will leave the coalition and form its own coalition. The process of evaluating utility, evaluateUtility(), is based on cooperative game theory and is explained in Section III-A. If a decision to stay in the coalition is made, the vehicle $V i$ will influence that coalition using InfluenceCoalition(). Note that it can only influence vehicles that are in its neighbourhood, $\mathrm{Ni}$. The details of influenceCoalition() method are presented in Section III-B. Once a decision to either remain in the coalition, or leave it is made, the agent will check, if it is part of a stable coalition. A stable coalition is defined as a coalition, which has agreed to a common speed for a period of time (in our simulations, we chose it to be 3 update cycles). If the coalition is stable then vehicle $V i$ can offer other vehicles in the neighbourhood to join that coalition using sendJoinOffer().

In coalition join decision algorithm, the vehicle $V i$ receives a coalition join offer from some neighboring vehicle $V j$ with coalition identifier $C j$. If vehicle $V i$ is already part of an existing coalition $C i$, it will compare the utility of both coalitions and join the offered coalition, $C j$, if it provides better utility. However, if the vehicle $V i$ is not part of any coalition, it will compare the utility of coalition $C j$ with its minimum utility, $M i$, and create a new self coalition, $C i$, if the offered coalition does not provide a better utility than its minimum requirement. Otherwise, it will join the offered coalition, $C j$.

These algorithms provide only the generic approach of the coalition formation process. The details of the utility evaluation and the influences between coalition members are presented in the following sections.

\section{A. Evaluating the utility of a coalition}

The utility of participating in a convoy depends on the goals of the driver. For example, if the prevailing goal is safety, the driver will participate in any convoy, provided that it is moving slower then the speed limit. If the goal is fastest possible traveling speed, the vehicle will not join any existing convoy. However, a new convoy might form from vehicles following the given vehicle.

An important observation is that the approach we take in this paper does not require the vehicles to use the same utility evaluation function. Once they join a convoy however, they need to have the same rules for evaluating influences.

We model the utility of a coalition for a vehicle as a function with values in the interval $[0,1]$ which depends on the following parameters:
$P i$ : $\quad$ Current speed of the vehicle $V i$
$S i j$ : Speed offered to vehicle $V i$
by coalition $C j$
$U i$ : $\quad$ Upper speed limit for vehicle $V i$
$L i$ : $\quad$ Lower speed limit for vehicle $V i$
$D i$ : $\quad$ Desired speed of vehicle $V i$
$M i$ : Minimum utility acceptable to vehicle $V i$ to remain in the coalition

Using these parameters, we can devise a number of utility functions. The function used in our experiments for a vehicle $V i$ to join coalition $C j$ is:

$$
U(i, C j)= \begin{cases}1-\frac{|D i-S i j|}{D i}-\lambda(j) \frac{|P i-S i j|}{P i} & \text { if } L i \geq S i j \leq U i \\ 0 & \text { otherwise }\end{cases}
$$

The cost factor, $\lambda(j) \frac{|P i-S i j|}{P i}$, is the cost of joining coalition $j$, and it is zero if $V i$ is currently member of the coalition. This factor reflects certain physical realities such as the need to accelerate or deccelerate to join a coalition, and itself is dependent on factors such as the difference between the speed of the convoy and the current speed of the vehicle. In addition, this factor allows us to introduce a certain "friction" in the behavior of the vehicles, reducing the number of defections and stabilizing the convoys. Experimentally, we found constant values of about 0.05-0.1 to be adequate for $\lambda(j)$.

\section{$B$. Influences between the members of the coalition}

Joining a convoy is not a purely logical operation. The vehicles in the convoy are reciprocally influencing each others' road behavior such that they are maintaining the desired formation. The input of these influences are based on data collected by the CDDs. Remarkably, stable convoys can be 
formed by knowing only the speed of the current vehicle. A better control can be maintained if the agent also has access to the distance of the neighboring vehicles. A single such measurement, such as the distance from the vehicle in front, is in practice sufficient. The ad-hoc network formed by the members of the coalition allows the vehicles to form a global picture of the convoy. This measurement can come from a radar-like device, or it can be inferred from the measurement of power of the wireless signal. The power of the wireless signal is, however, strongly dependent on the environment and needs to be used carefully.

In the following we discuss three influence strategies we tested.

1) Adjusting to the speed of the leader: In this case, the front vehicle of the convoy is considered the leader, and all the other vehicles in the coalition are adapting their speed to it. The advantage of this approach is that the coalitions are formed quickly and without the need of additional negotiation. Also there is no need of a distance measurement, except the implicit one provided by the fact that the vehicles are in the wireless range from each other. The disadvantages are that the speed of the convoy is dictated by its slowest vehicle, the only other choice of the member vehicles being to leave the convoy. This influence strategy alone is not able to adjust the following distances, altough in a practical deployment the driver can intervene through manual control if the vehicles come dangerously close to each other.

2) Average desired speed: In this case, the vehicles in the coalition adjust their speed to the average desired speed of the members. In general, this leads to a higher average utility of the coalition, for their members. Determining the average desired speed and forming a stable coalition can take several iterations. What is more, vehicles leaving or joining the coalition leads to a change in its speed. Thus, in every such occasion, the vehicles need to reevaluate their view of the utility of the coalition. If, for instance a "slow" vehicle joins the coalition, it will lower coalition speed. This in turn, might trigger a mass exodus of the other vehicles with higher desired speed. The resulting set of faster vehicles might create a coalition of their own, leading to a split of the convoy into a fast and a slow one. This approach, like the previous one, relies only on speed information, and it is not able to control the intervehicle distances.

3) Social potential fields: Social potential fields [3] form the basis of distributed behaviour control scheme. It is based on the idea of applying artificial forces among objects to keep them in group formation. These forces are inverse polynomial with the distance between the vehicles, and usually employ both attraction and repulsion forces. The formula we used for the force between two vehicles is:

$$
F(r)=\frac{-c 1}{r^{a 1}}+\frac{c 2}{r^{a 2}} \quad \text { where } \quad c 1, c 2 \geq 0, a 1>a 2>0
$$

We assumed that the forces are active only between the vehicles which are in communication range of each other. This approach presented the following advantages:

- The following distance between vehicles can be adjusted using the parameters $c 1, c 2, a 1$ and $a 2$.

- The coalition speed tends to converge towards the average speed of all the vehicles. Also, the change in speed is not sudden and takes place over a period of time.

The obvious difficulty of the approach is that it requires the existence of distance measurements.

We present the simulation results of each of these approaches in Section $\mathrm{V}$.

\section{IMPLEMENTATION}

Motes [2] are tiny computing devices with wireless networking capability. We carried out our experiments using Mica2 Mote Development Kit, running the TinyOS [4] programming environment and the nesC [5] programming language.

nesC resembles the syntax of the $\mathrm{C}$ language, while providing additional capabilities in the context of the TinyOS system, such as managing concurrency and promoting the use of components. Many low level functions, like sending packets to the serial port, radio communication or radio range specification have a component-based implementation. The TinyOS system also provides the TOSSIM simulation environment which allows us to debug the applications on a PC platform before deploying it on motes.

\section{A. Experimental setup}

Our experimental setup consisted of a desktop PC and several MICA2 motes. The desktop PC was used to develop the applications and simulate them with different number of nodes. Once the application was ready, it was deployed to the motes by uploading them through an interface board connected to the PC via serial cable. We found that the processing power of the motes are sufficient for the task, and even input/output problems were more related to the interfacing with the vehicles than with the capabilities of the motes. For instance, the only user interface feature on motes are three onboard LEDs. We used them to indicate whether the speed of the vehicle should increase or decrease, and left to the driver to adjust the cruise control system accordingly.

Figure 2 shows the components used in the implementation of our application. The SingleTimer component was used to fire timer events after given interval. The RadioCRCPacket component was used to send and receive packets over radio communication and it used UARTFramedPacket component to send packets over UART. It also used RandomLFSR component to generate random numbers (e.g. for coalition identifier) and the LedsC component to handle LEDs.

Each mote was initialized with a fixed node identifier and the current speed of the vehicle. They were programmed to periodically broadcast their node identifier, coalition identifier and current speed to all neighbouring nodes. This broadcast took place every $100 \mathrm{msec}$. The broadcast range was fixed using CC1000Control interface provided by RadioCRCPacket component. 


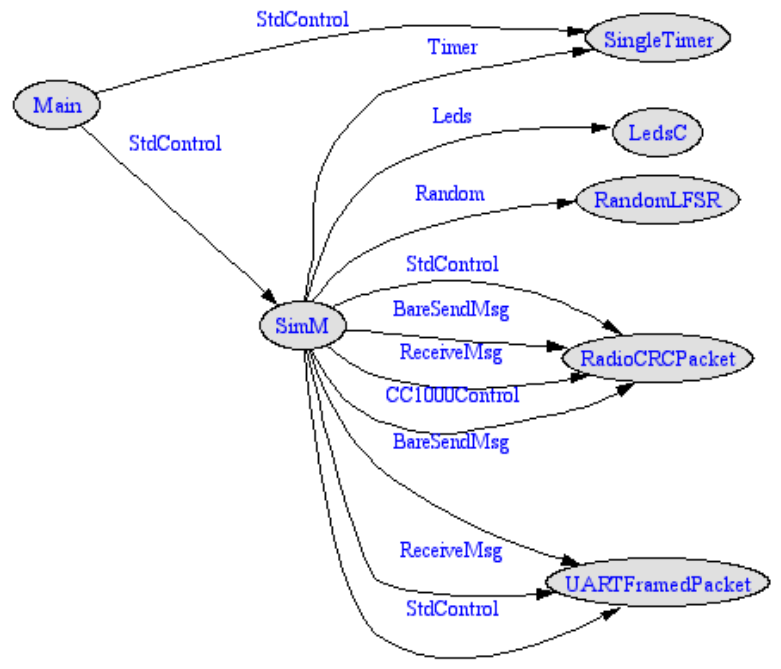

Fig. 2. Component diagram for convoy driving device application

While listening to incoming messages, each mote decided whether to join a coalition or not based on the utility function. If the incoming message was from the same coalition that the vehicle is part of, it used the message to adjust its own speed using the selected influence mechanism. If the new speed required acceleration, it turned on the red LED, while if breaking was required it turned on the yellow LED. If the current speed was equal to the coalitions adopted speed, the green LED was blinking. The current speed was sent over UART so that it could be inspected on attached PC via serial port. Figure 3 shows the deployed mote placed at the dashboard of the car as it is used to increase or decrease speed based on the indication from the LEDs.

\section{Simulation}

Our experimental setup showed that the presented algorithms can be used to form ad hoc coalitions with the purpose of convoy driving. To compare the results of the algorithms however, we need to perform more extensive experiments, involving various traffic conditions and larger number of vehicles. We performed these simulations using the YAES simulation framework [6].

The questions we planned to answer with these simulations are as follows:

- How do the algorithms behave in the presence of a dynamic set of vehicles?

- How are the three algorithms ("Adapting to the leader", "Average speed" and "Social Potential Fields") compare to each other?

- How effective are the algorithms in developing a smooth traffic condition?

- How does the approach scale with the number of vehicles?

- How robust is the approach in handling an onslaught of several kinds of traffic conditions?

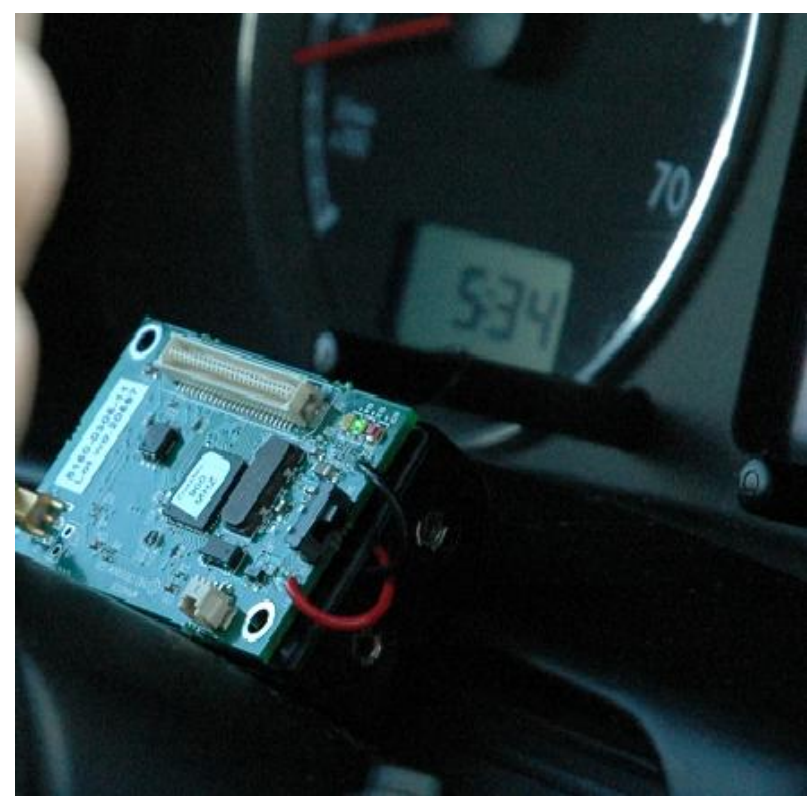

Fig. 3. Mote blinking to advise change in speed

\section{A. Comparing the influence mechanisms}

In the following simulation runs we are comparing the three choices for the influence mechanisms under various traffic conditions. To restrict the test only on the influence mechanism, we changed the join decision mechanism such that all vehicles in the test formed a single convoy. In our simulations we assumed the range of the wireless transmitter to be 20 meters.

The output of the simulation was the evolution of the speed and position of each vehicle. To achieve a better visualization of the configuration of the convoy, our position graphs represent the relative position of the vehicles in relation to a randomly chosen "lead". The dotted lines of the position graph represent the relative positions where the vehicles would be if without the coalition algorithms. The reason for this visualization approach is the fact that the relative movements of the vehicles are small compared with their common longitudinal movement, which would tend to dominate the absolute position plot.

1) Typical traffic conditions: Our first simulation assumes five vehicles on a highway moving with different speeds. The initial configuration is shown in the following table:

\begin{tabular}{|l|l|l|l|l|}
\hline ID & Position & Speed(m/s) & Low Speed & Hi Speed \\
\hline 1 & 130 & 18 & 14 & 26 \\
2 & 70 & 26 & 14 & 28 \\
3 & 100 & 25 & 14 & 26 \\
4 & 40 & 35 & 14 & 37 \\
5 & 0 & 35 & 14 & 37 \\
\hline
\end{tabular}

Figure 4 shows the results of "adapt speed to the leader" algorithm. We find that with this algorithm vehicles form coalitions quickly. It is important to note the radical speed changes which the vehicles need to make. in order to join the convoy, and the fact that the final speed of the convoy is the 
speed of the slowest vehicle.
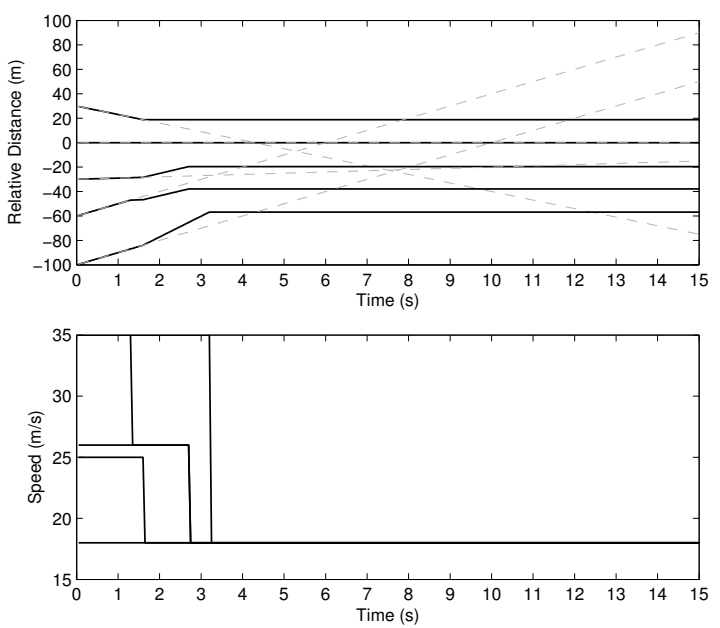

Fig. 4. Relative distance and speed variations with "adapt speed to the leader" influence

Figure 5 shows the results of the "average speed" influence algorithm. As expected, the speed changes are smaller in this case. However, the algorithm can introduce several speed changes over a poriod of time. The relative distance between vehicles, just like the previous case, is stabilizing around the maximum transmission range (i.e. $20 \mathrm{~m}$ ), as the vehicles quickly form coalitions as they enter each others communication range and agree upon average speed. As our simulation does not model the inevitable errors and fluctuations of the transmission range, the relative distance graph looks much smoother in this diagram than it would be in reality.
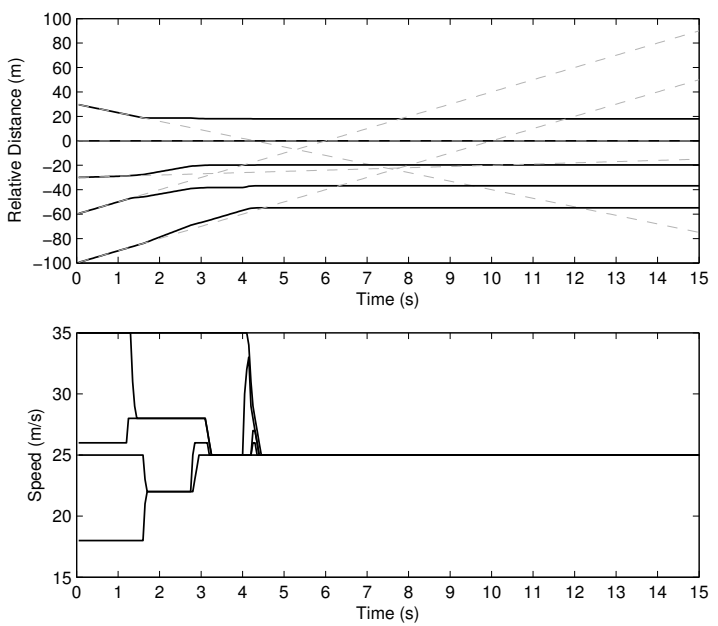

Fig. 5. Relative distance and speed variation with "average speed" influence

Figure 6 shows the results of social potential fields based influence algorithm. In this case, the speed changes are much smoother and it take place over a period of time. The equilibrium distance between the vehicles can be controlled using the parameters of the forces in the social potential field. In the simulation, parameters are set to achieve an equilibrium distance between vehicles at approximately 10 meters. We need to note that this value is robust for small disturbances, which will be automatically corrected by the algorithm. Also note that the resulting speed of the coalition will settle around the average speed of participating vehicles without the need of additional effort.
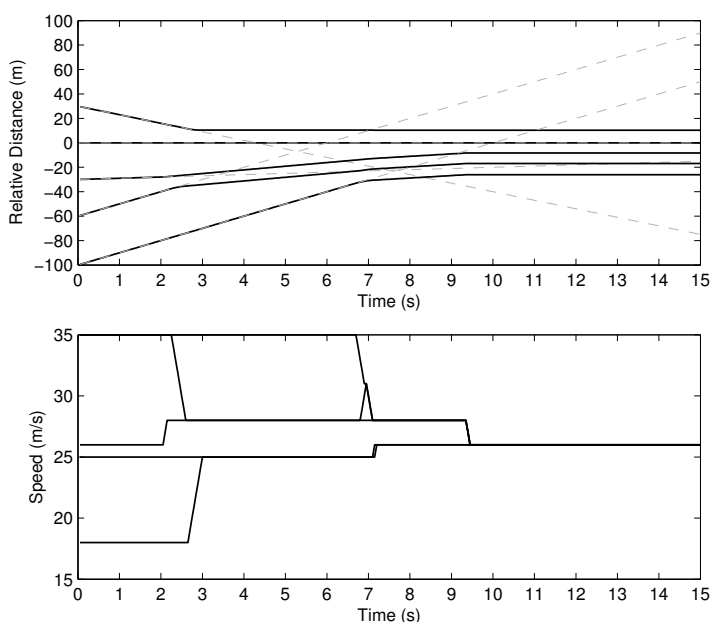

Fig. 6. Relative distance and speed variation with "social potential fields" influence in typical traffic conditions

2) Realistic traffic condition 1: This simulation run assumes a more realistic distribution of the different vehicle speeds. We assume the presence of different kinds of driving behaviours. Usually, on a highway some slow vehicles are followed by some moderately high speed vehicles and some very high speed vehicles. The following table shows the initial configuration of vehicles:

\begin{tabular}{|l|l|l|l|l|}
\hline ID & Position & Speed(m/s) & Low Speed & Hi Speed \\
\hline 1 & 170 & 18 & 14 & 40 \\
2 & 150 & 18 & 14 & 40 \\
3 & 90 & 24 & 14 & 40 \\
4 & 65 & 25 & 14 & 40 \\
5 & 20 & 40 & 14 & 40 \\
6 & 0 & 40 & 14 & 40 \\
\hline
\end{tabular}

This simulation assumed the "social potential fields" based influence algorithm. Figure 7 shows the results of the simulation. As expected, the vehicles tend to make coalitions and changes their speeds accordingly. The relative distance between vehicles also tends to be same within coalition. Note that multiple convoys are formed in this case. Also note the number of intersection points that would have occurred due to irregularities. Coalition formation reduces these intersections and thus smoothes out overall flow of vehicles. The speed of the coalition(s) lies around the average speed of participating vehicles. One note of caution is that in Figure.7, vehicles visually appear more closer than in Figure. 6 due to the change in graph scale. But actually they maintain the same distance of approaximately 10 meters. The same caution should be applied while interpreting graphs of other simulations. 

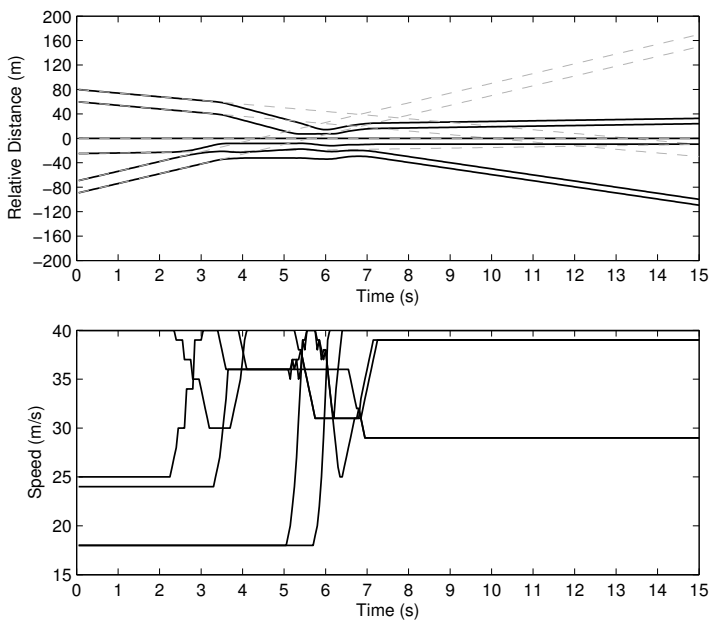

Fig. 7. Relative distance and speed variation with "social potential fields" influence in realistic traffic condition 1

3) Realistic traffic condition 2: This simulation shows a traffic condition where slow vehicles exist in between some moderate and high speed vehicles. The vehicle configuration is shown in the following table:

\begin{tabular}{|l|l|l|l|l|}
\hline ID & Position & Speed(m/s) & Low Speed & Hi Speed \\
\hline 1 & 170 & 18 & 14 & 26 \\
2 & 130 & 18 & 14 & 26 \\
3 & 110 & 22 & 14 & 28 \\
4 & 50 & 18 & 14 & 26 \\
5 & 20 & 31 & 14 & 37 \\
\hline
\end{tabular}

Figure 8 shows the results of this simulation. Again, the vehicles form coalition(s) where approximate distance between vehicles is 10 meters. The coalition speed also tends towards average speed of the vehicles. Note the effects of coalition merging. As fast moving vehicles approach a slow vehicle, they form a coalition. These different coalitions then merge into a single coalition as they approach each other.
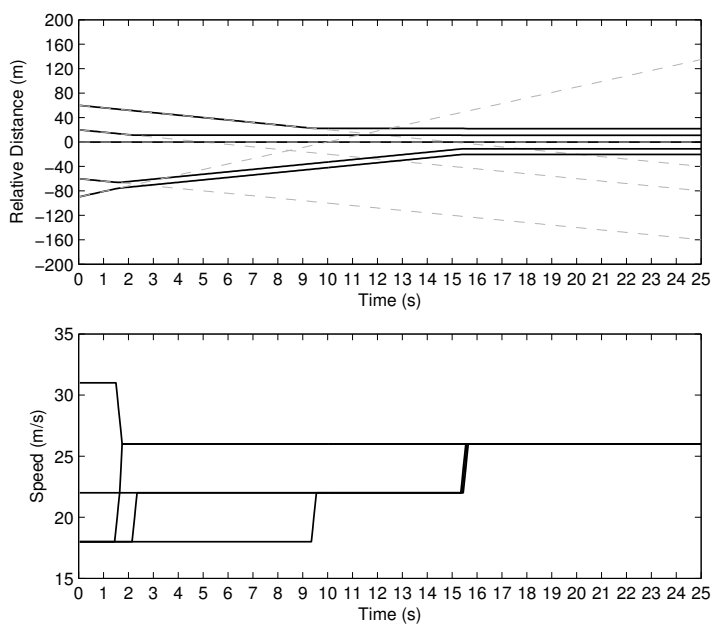

Fig. 8. Relative distance and speed variation with "social potential fields" influence in realistic traffic condition 2
4) Random traffic condition: In this case, we randomly selected current speed and speed limits of 10 vehicles and put them on the highway. Figure. 9 shows the results of this simulation. This simulation shows how a chaotic traffic can be transformed into a smooth traffic flow using "social potential fields" influence algorithm. The vehicles in the close proximity tend to form quick coalitions and the speed of these coalitions tends towards average speed of the participating vehicles.
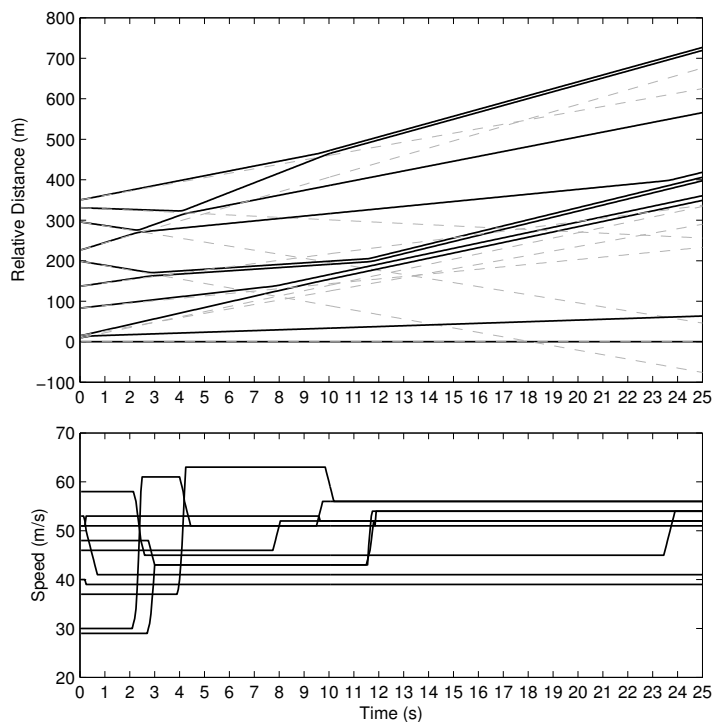

Fig. 9. Relative distance and speed variation with "social potential fields" influence in random traffic conditions

\section{B. Utility and influence mechanism simulation}

This simulation demonstrates the use of both utility function and influence mechanism. The vehicle configuration is shown in the following table:

\begin{tabular}{|l|l|l|l|}
\hline ID & Position & Speed(m/s) & Desired Speed(m/s) \\
\hline 1 & 180 & 17 & 24 \\
2 & 155 & 21 & 24 \\
3 & 120 & 24 & 40 \\
4 & 25 & 35 & 40 \\
5 & 8 & 36 & 40 \\
\hline
\end{tabular}

In this configuration, two fast moving vehicles in a coalition, approached a coalition of slow moving vehicles. Both vehicles that were in the fast coalition had high desired speed. So they showed not interest in joining the slow coalition. But one of the vehicle in slow moving coalition had high speed desirability. So as soon as it got an offer from the faster coalition, it joined it. The results are shown in the relative distance graph in Figure.10. This simulation shows how the utility and influence based approach can cause joining and abandonment of a coalition by a vehicle.

\section{RELATED WORK}

The work presented in this paper is related to a number of efforts in the development of vehicle control systems, ad-hoc networking protocols and team formation. 


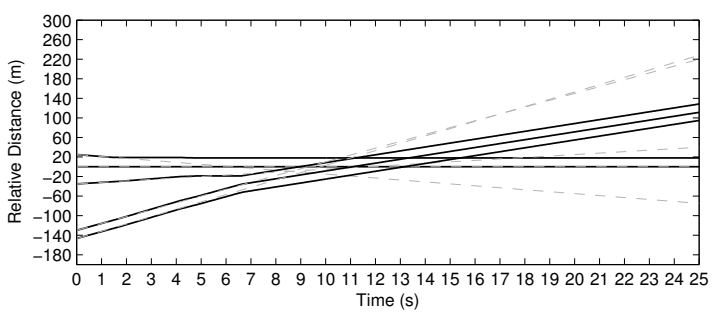

Fig. 10. Coalition joining and abandonment based on utility and influence mechanism

Convoy driving is an important concern for the transportation and automotive community. One of the approaches related to the one proposed in this paper are the Adaptive Cruise Control devices. Companies such as Daimler Chrysler and Jaguar have implemented and road tested prototypes of these devices. ACCs rely on measuring the distance from the previous vehicle through a radar, and they are controlling the accelerator, engine powertrain and vehicle brakes to maintain a desired time-gap to the vehicle ahead. These systems do not assume inter-vehicle communication capabilities. Marsden et. al [7] describe a set of simulations evaluating the benefit of such systems and summarize the literature concerning their adoption problems.

Most of these systems are based on radar-based measurements, although there are examples on work on fully automated convoy-driving based on computer vision as well [8].

PATH [9] is a research collaboration between UC Berkeley and California Department of Transportation. It carries out research to increase highway capacity and safety. Their main research focus is also towards automated vehicle control system and traffic management rather than inter-vehicle communication based systems.

The European Cartalk-2000 [10] project developed a specialized MAC protocol for intervehicle communication called Reliable R-ALOHA (RR-ALOHA). One of the future plans of the group is to apply it to "communication-based longitudinal control" which is largely equivalent to convoy driving. Briesemeister and Hommel [11] define a special multicast protocol suitable for inter-vehicle communication, where a multicast group is defined implicitly by location, speed, driving direction and time.

An approach using the JACK multi-agent framework for highway "platoon" formation is presented in [12]. The paper discusses various approaches for centralized and decentralized platoon formation and presents several control functions including a linear longitudinal control function and a neural network based approach.

\section{CONCLUSIONS}

Coalition formation on highways results in safer and coordinated traffic. Our paper present a flexible and distributed coalition formation scheme, which allows each vehicle to make its own autonomous decision to join a coalition and then have influence over it. We show the effects of these individual decisions in the evolution of a coalition structure.

Our utility function can be used by each vehicle to determine the coalition utility based on current environment and user defined set of parameters. We show how such an autonomous behaviour results in coalition joining and abandonment.

We explain the use of different influence mechanisms and demonstrate their basic characteristics using our simulation framework [6]. We show that quite reasonable coalitions can be formed with speed and distance information of neighbouring vehicles. The "social potential fields" based influence scheme can be used for not only forming coalitions but also to adjust inter-vehicle distance within a coalition. We also demonstrate the ease with which several coalitions merge to form a larger coalition.

Our mote based implementation demonstrates that we can easily adapt these ideas in practice. The motes are quite readily available in the market and very inexpensive to use. Moreover, they can be easily integrated with existing vehicle electronics system using available interfaces.

We therefore conclude that it is quite feasible to develop such coalition formation systems for commercial purposes. As modern vehicles provide a lot of driver assistance tools on board, the addition of coalition formation suggestions will be quite appealing to both vehicle consumers and transportation authorities.

\section{REFERENCES}

[1] N. Bellomo and M. Delitala, "On the mathematical theory of vehicular traffic flow I: Fluid dynamic and kinetic modelling," Mathematical Models and Methods in Applied Sciences, vol. 12, no. 2, pp. 1801-1843, 2002.

[2] J. Polastre, R. Szewczyk, C. Sharp, and D. Culler, "The mote revolution: Low power wireless sensor network devices," in Proceedings of Hot Chips 16: A Symposium on High Performance Chip, 2004.

[3] J. Reif and H. Wang, "Social potential fields: A distributed behavioral control for autonomous robots," in Proceedings of International Workshop on Algorithmic Foundations of Robotics (WAFR), 1995.

[4] "Tinyos webpage," URL http: / / www.tinyos.net.

[5] D. Gay, P. Levis, R. von Behren, M. Welsh, E. Brewer, and D. Culler, "The nesC language: A holistic approach to networked embedded systems," in Proceedings of ACM SIGPLAN Conference on Programming Language Design and Implementation, 2003.

[6] "YAES: yet another extensible simulator," URL http: / / netmoc.cpe. ucf.edu/Yaes/Yaes.html.

[7] G. Marsden, M. McDonald, and M. Brackstone, "Towards an understanding of adaptive cruise control," Journal of Transportation Research Part C: Emerging Technologies, vol. 9, pp. 33-51, 2001.

[8] H. Schneiderman, M. Nashman, A. J. Wavering, and R. Lumia, "Visionbased robotic convoy driving," vol. 8, no. 6, pp. 359-364, 1995.

[9] "California partners for advanced transit and highways," URL http: / / www . path. berkeley.edu.

[10] D. Reichardt, M. Miglietta, L. Moretti, P. Morsink, and W. Schulz, "Cartalk 2000 - safe and comfortable driving based upon inter-vehiclecommunication," in Proceedings of the IEEE Intelligent Vehicle Symposium (IV02), 2002.

[11] L. Briesemeister and G. Hommel, "Overcoming fragmentation in mobile ad hoc networks," Journal of Communications and Networks., vol. 2, no. 3, pp. 182-187, Sep 2000.

[12] S. Hallé and B. Chaib-draa, "Collaborative driving system using teamwork for platoon formations," in Proceedings of AAMAS-04 Workshop on Agents in Traffic and Transportation (ATT04), 2004. 